\title{
IN SILICO STUDY OF ARYL EUGENOL DERIVATIVES AS ANTI-COLORECTAL CANCER BY INDUCING OF APOPTOSIS
}

\author{
FADILAH FADILAH ${ }^{1,2 *}$, ARRY YANUAR ${ }^{1}$, ADE ARSIANTI $^{2}$, RETNOSARI ANDRAJATI ${ }^{1}$, \\ ERNI HERNAWATI PURWANINGSIH ${ }^{3}$
}

\begin{abstract}
${ }^{1}$ Department of Pharmacy, Faculty of Pharmacy, Universitas Indonesia, Depok Beji, Indonesia. ${ }^{2}$ Department of Medical Chemistry, Faculty of Medicine, University of Indonesia, JalanSalemba Raya 6 Jakarta 10430, Indonesia. ${ }^{3}$ Departement of Pharmacy, Faculty of Medicine, Universitas Indonesia, Salemba Raya 4 Jakarta 10430, Indonesia. Email: fadilah81@gmail.com
\end{abstract}

Received: 13 July 2017, Revised and Accepted: 15 September 2017

ABSTRACT

Objective: Apoptosis is one method the body uses to get rid of unneeded or abnormal cells, but cancer cells have strategies to avoid apoptosis. Apoptosis inducers can get around these strategies to cause the death of cancer cells.

Methods: We screened some derivatives aryl eugenol based on their interactions with Bcl-2 in many cancer tissues, using computer software applications (in silico method) to determine the best compounds. The docking experiment on Bcl-2 (Protein Data Bank ID 4LXD) was carried out by suitably positioning the energy-minimized ligand in the active site while carefully monitoring non-bonded interactions of the ligand enzyme.

Results: The resulting ligand-receptor complex was docked using the Autodock Vina software. Docking results based free binding energy, EUGACl (21), EUASABr (17), EUGEABr (19), and EUASACL (17), has the lowest binding energy than navitoclax and binds significantly to BCL 2. In silico ADMET predictions revealed that except SA, ASA, and GEA, all other compounds had minimal toxic effects and had good absorption as well as solubility characteristics.

Conclusion: These compounds of aryl eugenol $(17,19$, and 21$)$ may serve as a potential lead compound for developing new anticancer as apoptosis inducers.

Keywords: In silico, Aryl eugenol derivatives, Apoptosis inducer, Docking simulation, Drug-likeness.

(C) 2017 The Authors. Published by Innovare Academic Sciences Pvt Ltd. This is an open access article under the CC BY license (http://creativecommons. org/licenses/by/4. 0/) DOI: http://dx.doi.org/10.22159/ajpcr.2017.v10i12.21233

\section{INTRODUCTION}

Cancer is one of the health problems in the world and a leading cause of death after heart disease [1]. Data satistics show that many people who are diagnosed and die from cancer each year, by the 2014 , the number of people who died after being diagnosed with cancer reached 14.5 million and is expected to increase nearly 19 million in 2024 [2]. It requires serious therapy. Targeted cancer therapies include designing compounds that can interfere with or inhibit the growth of tumors. Several therapies are done including gene expression therapy, inhibitor signal transduction, modulator and inducer of apoptosis, immunotherapy, and toxin delivery molecules [2]. Apoptosis is programed cell death characterized by cell membrane blebbing, chromatin condensation, and chromosomal DNA fragmentation. Apoptosis as a form the body's way of getting rid of cells that are not needed or are not normal, but cancer cells have strategies to avoid this apoptosis. The presence of apoptosis inducers can cause the death of cancer cells [2]. This is basically influenced by the role of Bcl-2 protein family [3-5]. Bcl-2-family proteins regulate cell death and proliferation $[6,7]$, which are two processes dysregulation during oncogenic transformation.

Bcl-2 family of proteins is a key role in controlling apoptosis in the mitochondria and also in the control of cell proliferation. High levels of Bcl-2 family of proteins that have been studied related to the proliferation of different for each cancer [8,9]. The molecular mechanisms in suppressing apoptosis by tumor cells that are affected will cause resistance apoptosis, in which the tumor suppressor gene p53 $[10,11]$ forming a B-cell lymphoma that expression of Bcl-2 will be excessive. This proves that cancer is caused by a failure of cell death [12]. Over the past decade, there was a lot of progress in the discovery of promising new cancer therapies. This new therapy apoptosis of them tried to prime the machine that acts as apoptosis-inducing agent, and this method is used against cancers that are resistant to conventional treatment [13]. Many studies have explained that the targeted therapy is potentially for apoptosis [14] among them focus on Bcl-2 family of proteins [15]. BH3 domain of Bcl-2 consisting between 14 until 24 amino acid sequences. This sequences can be synthesized, which is pharmacologically active molecules which have a pro-apoptotic role in the cell. In addition, several small molecule as inhibitors Bcl-2 such as HA14-1, obatoclax, gossypol. Others natural compounds from phenolic, polyphenol and phenylpropanoids proven to be able to inhibit Bcl-2. Manal et al. conducted that phenolic compounds such as caffeic acid and ferulic acid had promising antitumor activity with $\mathrm{IC}_{50}$ values of 6 and $10 \mu \mathrm{g} / \mathrm{ml}$, respectively [16].

The mechanism of small molecules such as polyphenols can inhibit the binding of Bcl-2, Bcl-xL, and Bax. ABT-737 has similarities with BH3 protein docking and has hydrophobic properties of the anti-apoptotic protein, thus disabling the capacity to pro-apoptotic protein [17]. Polyphenol (rhein) inhibited cancer cell proliferation, upregulated thep53, Bax, Casp-3, and -9 genes and downregulated the Bcl-2 gene and ultimately leads to genomic DNA fragmentation [18]. The approach is widely used in the search for small molecule compounds as using a database using computational tools mostly done rather than directly screening, computational method is known as virtual screening [19]. However, the assessment of phenylpropanoids which has a functional derivative of aryl groups for BCL 2 is still unknown. In this work, we are using virtual platforms screening based on protein structure (structure based) [20,21] and determining the drug-likeness using rules Lipinski [22] where Lipinski is complementary to identify compounds 
derivatives biaryl eugenol potential. The freshness of this research is to design a potential eugenol biaryl derivative that is structurally modified as Bcl-2 inhibitor. The purpose of this study was to investigate the potential eugenol aryl derivatives as inhibitors of Bcl-2 using a molecular docking approach. While energy-based docking schemes are based on having knowledge of the approximate positioning of the ligand in the receptor active site, the shape-based method is based on the assumption that the molecular surfaces of the receptor and the ligand need to match, if the molecules are to bind to each other with high affinity.

\section{METHODS}

Three-dimensional structure building and all modeling were performed software tool installed on Lenovo desktop workstations equipped with a dual $2.0 \mathrm{GHz}$ Intel Xeon processor running the Windows operating system.

\section{Aryl eugenol derivatives preparation}

The derivative structures were generated by Chem Draw Ultra 12.0 [23] for the molecular docking experiments, and their conformational energy was minimized using MMFF94 force field. Fourteen molecules of aryl eugenol derivatives were designed by substituting the group (R1, $\mathrm{R} 2, \mathrm{R} 3$, and R4) positions of aromatic group. The molecule structures are depicted in Fig. 1.

\section{Drug-likeliness evaluation}

The drug-likeliness properties of the selected compounds were investigated with the help of Lipinski drug filter under Chemicalize (Chem Axon) [24] and Molsoft [25] platforms. These physicochemical properties are important for developing the drug candidate in every stage from design, synthesis, and biological activity test to preclinical study. Lipinski rule of five is a rule of the thumb to evaluate drug-likeliness or determine if a chemical compound with a certain pharmacological and biological activity has properties that would make it likely orally active drug in humans.

\section{Protein preparation}

The crystal structure of the target protein was retrieved from Protein Data Bank (PDB) (4LXDJ), and minimization of the protein was generated by Python Molecular Viewer 1.5.6cr3. The protocol prepares the protein by inserting the added partial charges using Gasteiger method, add polar hydrogens in the protein and residual materials, such as water and ligand molecules, are removed before minimization.

\section{Active site prediction}

The minimized protein is further taken for binding site detection which will be very useful in the active site. This study has been used to know the important residues in the target protein which are responsible for ligand binding, present in the active site under active site prediction [26].

\section{Molecular docking simulation of Bcl-2-Aryl eugenol derivatives}

The preparative protein and ligand coordinates were saved as PDB files. The 3D structure (PDB 4LXD) was taken from the Research Collaboratory for Structural Bioinformatics PDB (http://www.rcsb. org/pdb) [27]. Molecular docking experiment is performed using Autodock Vina program (Vina, The Scripps Institute) to dock the aryl eugenol derivatives to the binding site of the Bcl-2 [28]. The Autodock Vina tools is used to add partial charges using Gasteiger method and to arrange the polar hydrogens in the protein. Energy minimizations

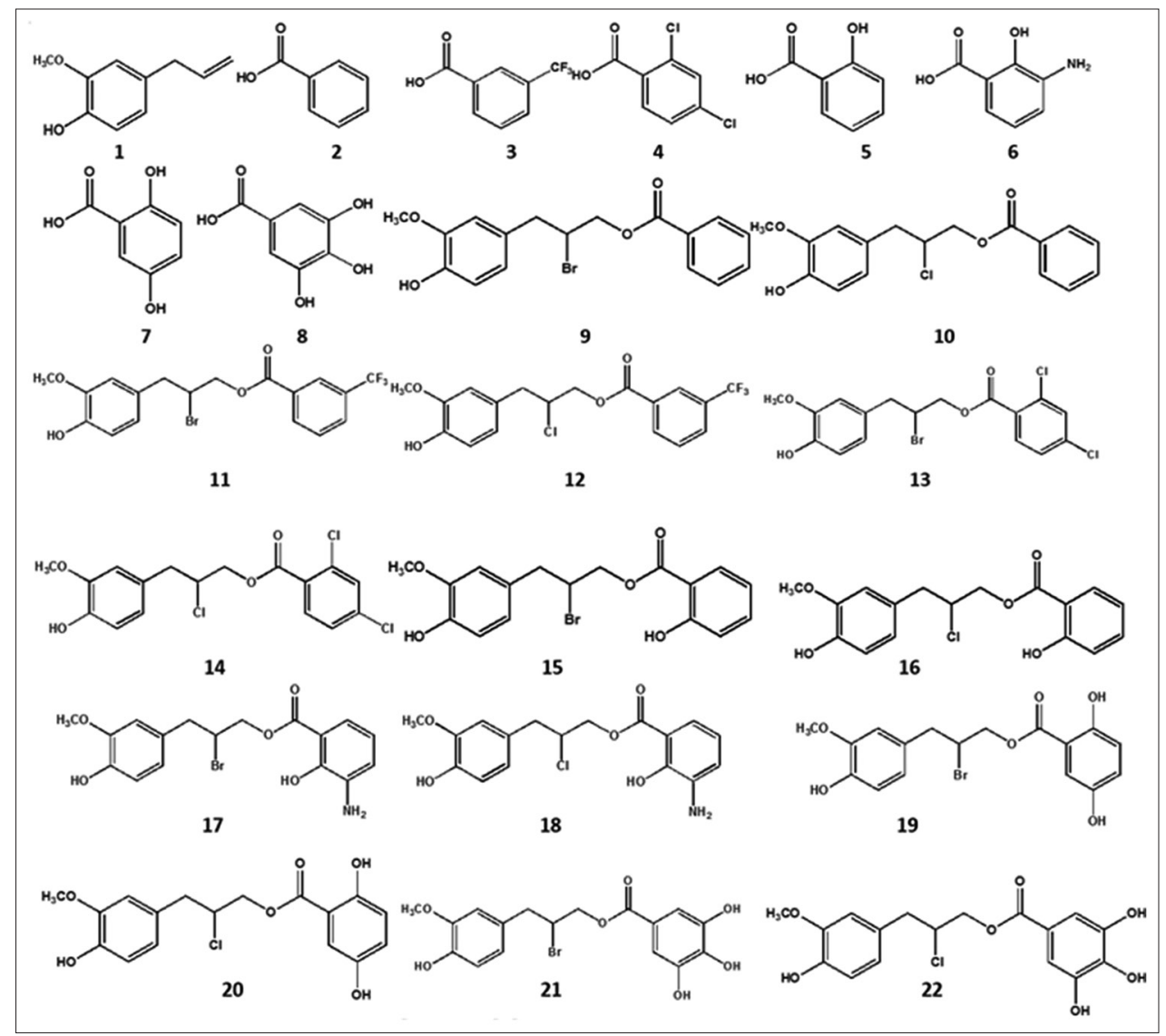

Fig. 1: Aryl eugenol derivatives. Molecule with number 1-8 is compounds from a natural product, molecule number 9-22 are compounds coupling between eugenol 1 and aryl group from compounds no 2-8. Aryl group from compounds number 9-12 were designed with addition with halogen $\mathrm{Br}$ and $\mathrm{Cl}$ 
were performed for 1000 iterations until reaching a convergence and the conjugate gradient algorithm with a convergence criterion of $0.01 \mathrm{kcal} /(\mathrm{mol} \mathrm{A})$. The ligands are set to have flexible torsion angles at all rotatable bonds, while the protein is prepared as a rigid structure. Both protein and ligand are saved as output pdbqt files. For specific docking of ligand aryl eugenol derivatives onto the BCL2 protein, the grid box volume was adjusted to $40 \times 40 \times 40 \AA$ in the $\mathrm{X}, \mathrm{Y}$, and $\mathrm{Z}$ axes, respectively, with grid-sizes have a space up to $1 \AA$. Autodock Vina employs an idealized active site ligand as a target to generate putative poses of molecules.

\section{Docking analysis of Bcl-2-aryl eugenol derivatives}

The binding energy values were calculated based on the total intermolecular energies (kj/mol) including hydrogen bond energy, Van Der Waals energy, desolvation energy, and electrostatic energy. On the other hand, analysis of screening compounds was based on the energy variation, due to the formation of the ligand-receptor structure, it is given by the binding constant and the Gibbs free energy $(\Delta G)$ values. Prediction of the binding energy is performed by evaluating the most important physical-chemical phenomena involved in ligand-receptor binding, including conformation of the structure and hydrogen bonding interaction between compounds and the target protein.

\section{RESULTS AND DISCUSSION}

In assessing the physicochemical of aryl eugenol derivatives, we evaluate the drug-likeness of derivative compounds using Chemicalize platform. This is a crucial parameter in drug development since it impacts both properties and target affinity of drug candidates. Drug-likeness indices are inherently limited tools. Drug-likeness can be estimated for any molecule and does not evaluate the actual specific effect that the drug achieves (biological activity). Simple rules are not always accurate and may unnecessarily limit the chemical space to search: Many best-selling drugs have features that cause them to score low on various drug-likeness indices [29]. Table 1 depicts the drug-likeness properties of test compounds with least binding energies predicted using Molsoft. The Molsoft tool measures the log P value (logarithm of compound's partition coefficient between octanol and water) which is a well-established measure of the compound's hydrophilicity. Higher $\log \mathrm{P}$ value indicates lower hydrophilicity, and thus, poor absorption and permeation. A lower molecular weight would again enhance the absorption rate, and thus, most of the drugs are tried to be kept at the lowest possible molecular weight [29]. In this study, all compounds have $\log \mathrm{P}$ values ranging from -0.833 to 4.894 . Topological polar surface area (TPSA) indicates the surface belonging to polar atoms in the compound.

An increased TPSA is associated with diminished membrane permeability, and compounds with higher TPSA were better substrates for p-glycoprotein (responsible for drug efflux from cell). Thus, comparing the compounds, lower TPSA was favorable for druglike property. It was also predicted that a molecule with better CNS penetration should have lower TPSA value [30]. All compounds have TPSA values ranging from 29.460 to 116.450 . In general, an orally active drug has no more than one violation according to Lipinski's rule of five, as followed criteria: Molecular weight: The smaller the better, because diffusion is directly affected [31].

The great majority of drugs on the market have molecular weights between 200 and 600 Daltons, and particularly <500 [32]; they belong to the group of small molecules. From in silico drug-likeness prediction along with further ADME which help in accelerating the discovery of new targets and ultimately lead to compounds with predicted biological activity. Results showed that all compound has followed Lipinski's rule of five. Based on the receptor cavity method using "eraser algorithm," we identified 5 active sites for the target protein the active site of protein BCL 2 has been depicted in Fig. 2.

The amino acids of the first site were selected as the active site for docking study has been depicted in (Fig. 3a). Number of amino acids are 26 (Gln40, Glu41, Arg43, Gln44, Val47, Arg51, Arg108, Asp109, Arg112, Glu116); (Glu114, Gln118); (Glu41, Gln44, Glu45, Asp48); (Gln118, Leu119, His120, Leu121, Thr122, Thr125, Gly125, Gly128, Arg128, Thr132).

Design a series of aryl eugenol derivatives molecule of 9-22 with diverse aryl moieties, a computer-aided molecular modeling study was carried out within the apoptosis regulator BCL2 of the high-resolution crystal structure (resolution $=2.1 \AA$ ) of the apoptosis regulator BCL 2 (PDB 5JSN). The protein crystal structure was retrieved from the Research Collaboratory for Structural Bioinformatics Protein Data Bank. The structure of the apoptosis regulator BCL 2 was used as a reference ligand along the docking and modeling studies.

Table 1: The physicochemical and drug-likeness of aryl eugenol derivatives

\begin{tabular}{|c|c|c|c|c|c|c|}
\hline Molecule & MW & $\log P$ & TPSA & HBD & HBA & Lipinski's \\
\hline Eugenol (Eu) & 164.204 & 2.129 & 29.460 & 1 & 2 & Yes \\
\hline Benzoic acid (BA) & 121.155 & 0.050 & 40.130 & 0 & 2 & Yes \\
\hline Meta tri fluoro methyl (TFB) & 189.112 & 1.380 & 40.130 & 0 & 2 & Yes \\
\hline 2,4 dichloro benzoic acid (CBA) & 190.005 & 1.357 & 40.130 & 0 & 2 & Yes \\
\hline Salicylic acid (SA) & 137.114 & -0.244 & 60.360 & 1 & 3 & Yes \\
\hline ASA & 152.129 & -0.662 & 86.380 & 2 & 3 & Yes \\
\hline Gentisic acid (GEA) & 153.113 & -0.539 & 80.590 & 2 & 4 & Yes \\
\hline EuBA-Br & 365.223 & 3.564 & 55.760 & 1 & 3 & Yes \\
\hline EuBA-Cl & 320.772 & 3.408 & 55.760 & 1 & 3 & Yes \\
\hline EuTFB-Br & 433.220 & 4.894 & 55.760 & 1 & 3 & Yes \\
\hline EuTFB-Cl & 388.769 & 4.738 & 55.760 & 1 & 3 & Yes \\
\hline EuCBA-Br & 434.113 & 4.871 & 55.760 & 1 & 3 & Yes \\
\hline EuCBA-Cl & 389.662 & 4.714 & 55.760 & 1 & 3 & Yes \\
\hline EuSA-Br & 381.222 & 3.269 & 75.990 & 2 & 4 & Yes \\
\hline EuSA-Cl & 336.771 & 3.113 & 75.990 & 2 & 4 & Yes \\
\hline EuGEA-Br & 397.221 & 2.975 & 96.220 & 3 & 5 & Yes \\
\hline EuGEA-Cl & 352.770 & 2.819 & 96.220 & 3 & 5 & Yes \\
\hline EuGA-Br & 413.220 & 2.681 & 116.450 & 4 & 6 & Yes \\
\hline EuGA-Cl & 368.769 & 2.524 & 116.450 & 4 & 6 & Yes \\
\hline Navitoclax & 698.241 & 3.998 & 133.080 & 4 & 8 & No \\
\hline
\end{tabular}

R05: Rule of five, TPSA: Total polar surface area, HBD: Hydrogen bond donor, HBA: Hydrogen bond acceptors. ASA: Aminosalicylic acid, GA: Gallic acid, TPSA: Topological polar surface area 
Binding energy is the primary parameter which is generated as a result of molecular docking. It gives us the idea of strength and affinity of the interaction between the ligand and the receptor. The greater the binding energy is the weaker the interaction and vice versa. Thus, during any docking study, we intend to look for the ligand which displays the least binding energy, thus the best affinity among the test molecules. Docking study of aryl eugenol derivatives was showed that EUGACl, EUASABr, EUGEABr, and EUASACL have lowest binding energy with value $-14.2625,13.0988,13.0828$, and $12.8963 \mathrm{kcal} /$ $\mathrm{mol}$, respectively, than navitoclax with value $-12.7657 \mathrm{kcal} / \mathrm{mol}$ was much higher than four derivatives, as found in our study; thus EUGACl displayed much better binding than all derivetives and the control molecule. The binding energies of the test ligands and the control have been depicted in Fig. 2.

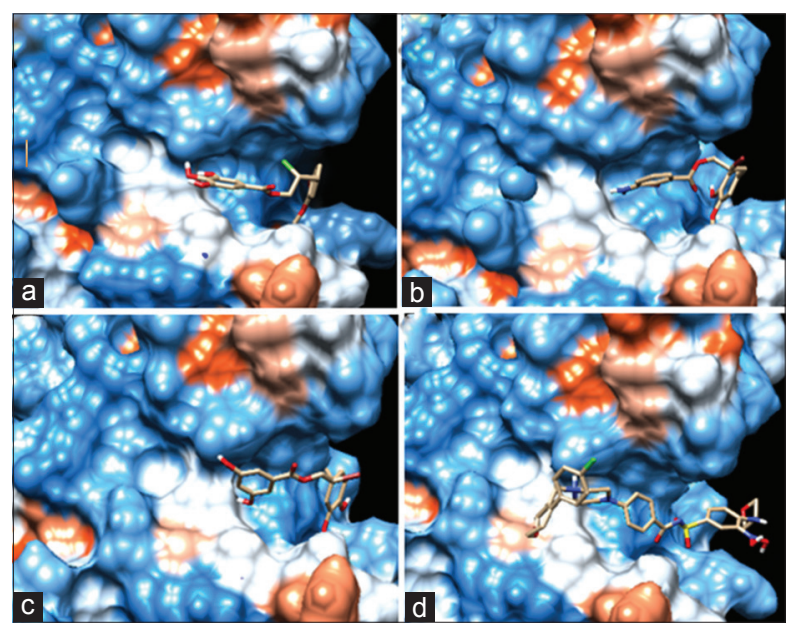

Fig. 2: The binding interactions of complexes into the cavity, white arrow are hydrogen bonding of complexes. Complex of 21 (EUGABr) have 3 hydroxyl group with BCL2 (a). Complex of 17 (EUASABr) have 1 hydroxy and 1 amino group with BCL2 (b) Complex of 19 (EUGEABr) have 2 hydroxy with BCL2 (c). Complex of navitoclax with BCL2 (d)
In silico docking indicated many interactions with active site of BCL 2 . The best score from the best pose for each compound was taken and compound to the scores of the other compounds. The compounds which show highest negative $\Delta \mathrm{G}(\mathrm{kcal} / \mathrm{mol})$ score shows that it has the capacity to bind strongly with the protein. Docked conformers of all the designed molecules were analyzed for the presence of similar interactions (Fig. 3b). In compounds 22, the presence of three of hydroxyl phenyl and $\mathrm{Br}$ halogen substitutions found to push the hydrophilic head portion toward the hydrophobic region and orient differently in the pocket. Due to this, the H-bonding interaction of BCL 2 with Glu45, Thr132, Glu41, His120, Arg129, Arg129, and Thr132 was totally absent (Fig. 2 and Table 2).

In case of compounds 17 and 18, have a hydroxyl and amine group in ring benzene, orientation greatly varies in comparison with compound 22 , and this compound reversed their orientation and could able to establish to H-bonding interaction with Asp48. Compounds 19 having two hydroxyl and $\mathrm{Br}$ Halogen in ring benzene due to their smaller size could able to position side chain carbonyl oxygen and hydroxyl group of BCL 2 in such a way to establish H-bonding interaction with Thr132, Glu45, His120, His120, Arg129, and Thr132. The three major interaction energies (VdW, ES, and HB) of these four compounds were found to be same with navitoclax (Table 2). Increasing the hydroxyl group (compounds 22 and 19) resulted in conformation quite similar

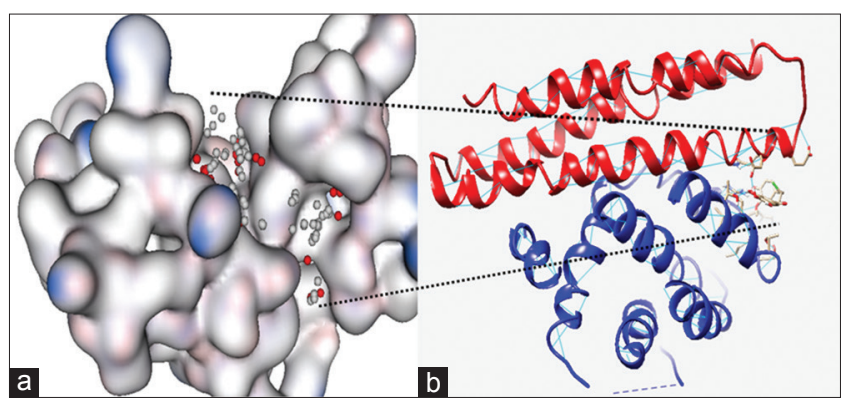

Fig. 3: Three dimension of regulator BCL 2. Active site analysis of regulator apoptosis BCL 2 (a), docking conformation of complexes aryl eugenol with regulator BCL 2 (b)

Table 2: Docking results of aryl eugenol derivatives-Bcl2

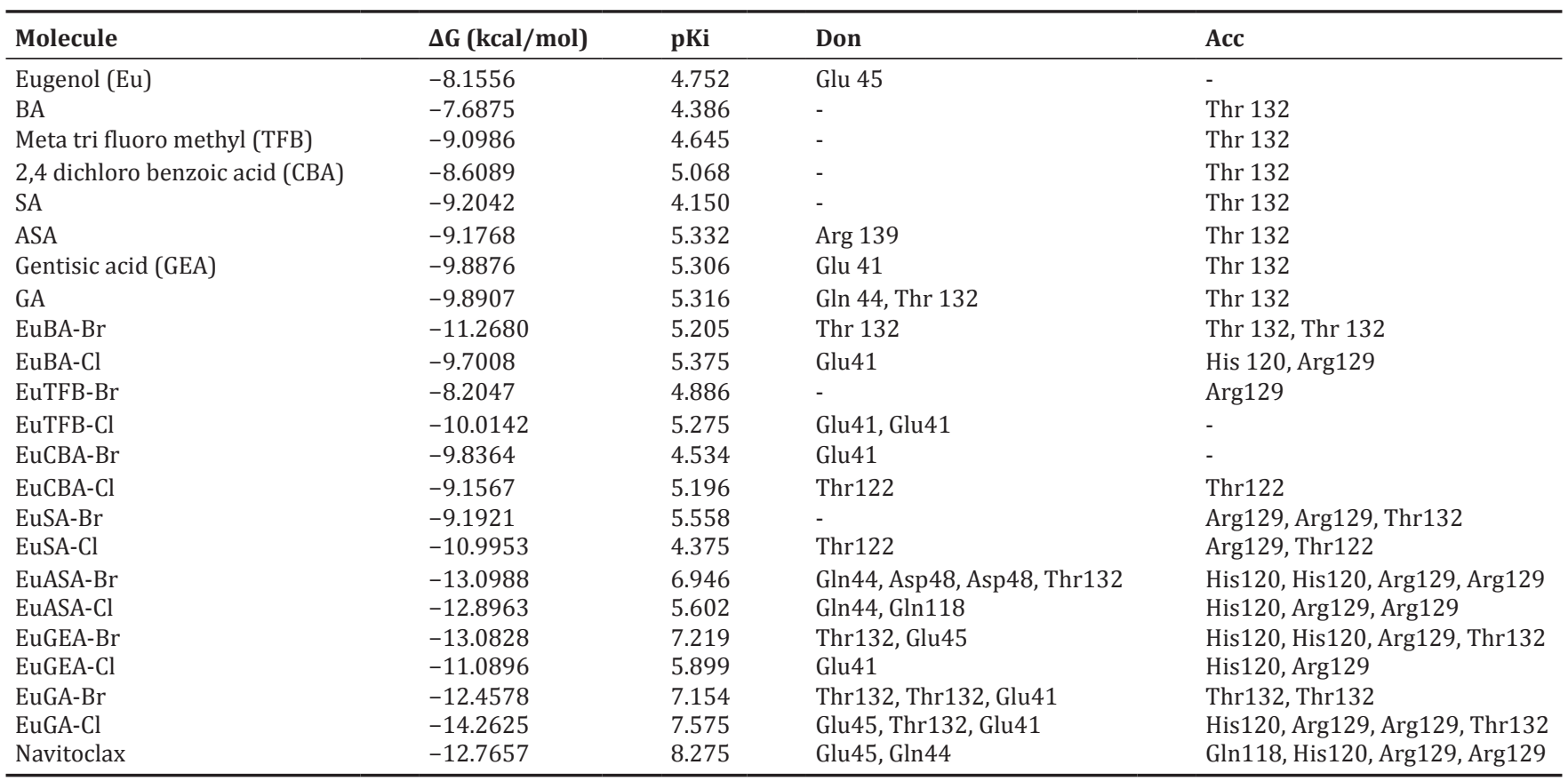

BA: Benzoic acid, ASA: Amino salicylic acid, SA: Salicylic acid, GA: Gallic acid 
to the one having an amino group (compound 17). Thus, compound 22, 17 , and 19 are promising candidates for new anticancer as apoptosis inducer agents and should be considered as the lead compounds in the next synthesis project.

\section{CONCLUSION}

In summary, the introduction of a hydroxyl functional group on aromatic ring improved the interaction with reversed orientation. This has made the phenyl ring portion to behave as hydrophilic head while pushing the active site of BCL 2. Through this study, we proposed a new class of aryl eugenol targeting BCL2 regulator apoptosis for the treatment of anticancer.

\section{ACKNOWLEDGMENTS}

The authors are thankful to Universitas Indonesia (UI), Indonesia, for providing facility, space, and resources for this work. The study was partly supported by a Research Grant from PITTA 2016.

\section{REFERENCES}

1. WHO. Available from: http://www.who.int/cancer/en.

2. Available from: https://www.cancer.gov/understanding/statistics

3. Czabotar PE, Lessene G, Strasser A, Adams JM. Control of apoptosis by the BCL-2 protein family: Implications for physiology and therapy. Nat Rev Mol Cell Biol 2014;15(1):49-63.

4. Renault TT, Chipuk JE. Death upon a kiss: Mitochondrial outer membrane composition and organelle communication govern sensitivity to BAK/BAX-dependent apoptosis. Chem Biol 2014;21(1):114-23.

5. Lindner AU, Concannon CG, Boukes GJ, Cannon MD, Llambi F, Ryan D, et al. Systems analysis of BCL2 protein family interactions establishes a model to predict responses to chemotherapy. Cancer Res 2013;73(2):519-28.

6. Vaux DL, Cory S, Adams JM. Bcl-2 gene promotes haemopoietic cell survival and cooperates with c-myc to immortalize pre-B cells. Nature 1988;335(6184):440-2

7. Bonnefoy-Berard N, Aouacheria A, Verschelde C, Quemeneur L, Marçais A, Marvel J. Control of proliferation by Bcl-2 family members. Biochim Biophys Acta 2004;1644(2-3):159-68.

8. Dai H, Meng W, Kaufmann SH. BCL 2 family, mitochondrial apoptosis and beyond. Cancer Transl Med 2016;2:7-20.

9. Tiwari P, Khan MJ. Molecular and computational studies on apoptotic pathway regulator, Bcl-2 gene from breast cancer cell line MCF-7. Indian J Pharm Sci 2016;78(1):87-93.

10. Marvel J, Perkins GR, Lopez Rivas A, Collins MK. Growth factor starvation of bcl-2 overexpressing murine bone marrow cells induced refractoriness to IL-3 stimulation of proliferation. Oncogene 1994;9(4):1117-22

11. Miyashita T, Krajewski S, Krajewska M, Wang HG, Lin HK, Liebermann DA, et al. Tumor suppressor p53 is a regulator of bcl-2 and bax gene expression in vitro and in vivo. Oncogene 1994;9(4):1799-805.

12. Vaux DL. Immunopathology of apoptosis - Introduction and overview. Springer Semin Immunopathol 1998;19(3):271-8.
13. Meiler J, Schuler M. Therapeutic targeting of apoptotic pathways in cancer. Curr Drug Targets 2006;7(10):1361-9.

14. Giménez-Bonafé P, Tortosa A, Pérez-Tomás R. Overcoming drug resistance by enhancing apoptosis of tumor cells. Curr Cancer Drug Targets 2009;9(3):320-40.

15. Delbridge AR, Grabow S, Strasser A, Vaux DL. Thirty years of BCL-2: Translating cell death discoveries into novel cancer therapies. Nat Rev Cancer 2016;16(2):99-109.

16. Hamed MM, Mohamed MA, Ahmed WS. Antioxidant potential, antitumor activity and phenolic profile dactyliferalinn. Int J Pharm Pharm Sci 2017;9:130-6.

17. Sharad V, Amit A, Abha M. Complex disruption effect of natural polyphenols on bcl-2-bax: molecular dynamics simulation and essential dynamics study. J Biomol Struct Dyn 2015;33:1094-106.

18. Fatlawi AA, Zafaryab MD, Irshad MD, Ahmad I, Kazim Z, Ahmad A, et al. Rhein induced cell death and apoptosis thought caspase dependent and associated with modulation of P53, BCL-2/BAX ratio in human cell lines. Int J Pharm Pharm Sci 2014;6:515-9.

19. Schneider G. Virtual screening: An endless staircase? Nat Rev Drug Discov 2010;9(4):273-6.

20. Schneider G. Virtual screening: An endless staircase? Nat Rev Drug Discov 2010;9(4):273-6.

21. Vyas VK, Gupta N, Ghate M, Patel S. Design, synthesis, pharmacological evaluation and in silico ADMET prediction of novel substituted Benz imidazole derivatives as angiotensin II-AT1 receptor antagonists based on predictive 3D QSAR models. SAR QSAR Environ Res 2014;25(2):117-46.

22. Zhang Y, Liu H, Jiao Y, Yuan H, Wang F, Lu S, et al. De novo design of N-(pyridin-4-ylmethyl) aniline derivatives as KDR inhibitors: 3D-QSAR, molecular fragment replacement, protein-ligand interaction fingerprint, and ADMET prediction. Mol Divers 2012;16(4):787-802.

23. Nogara PA, Saraiva Rde A, Caeran Bueno D, Lissner LJ, Lenz Dalla Corte C, Braga MM, et al. Virtual screening of acetyl cholinesterase inhibitors using the lipinski's rule of five and ZINC databank. Biomed Res Int 2015;2015:870389

24. Cousins KR. Computer review of chem draw ultra 12.0. J Am Chem Soc 2011;133(21):8388.

25. Southan C, Stracz A. Extracting and connecting chemical structures from text sources using chemicalize.org. J Cheminform 2013;5(1):20.

26. Available from: http://www.molsoft.com/mprop.

27. Available from: http://www.scfbio-iitd.res.in/dock/ActiveSite.jsp.

28. Berger S, Procko E, Margineantu D, Lee EF. Computationally designed high specificity inhibitors delineate the roles of BCL2 family proteins in cancer. Elife 2016;5. pii: E20352.

29. Trott O, Olson AJ. AutoDock Vina: Improving the speed and accuracy of docking with a new scoring function, efficient optimization, and multithreading. J Comput Chem 2010;31(2):455-61.

30. Available from: http://www.pipeline.corante.com/archives/2012/03/26/ whats_the_ugliest_drug_or_the_ugliest_drug_candidate.php.

31. Blake JF. Chemoinformatics - Predicting the physicochemical properties of 'drug-like' molecules. Curr Opin Biotechnol 2000;11(1):104-7.

32. Lipinski CA, Lombardo F, Dominy BW, Feeney PJ. Experimental and computational approaches to estimate solubility and permeability in drug discovery and development settings. Adv Drug Deliv Rev $2001 ; 46(1-3): 3-26$ 